\title{
A Rare Case of Invasive Endometriosis of the Rectum, Presenting as a Colonic Obstruction
}

\author{
Elias Fakhoury, d, Danon Garrido ${ }^{\mathrm{b}}$, Michael A. Lopez ${ }^{\mathrm{c}}$, \\ Miguel A. Lopez-Viego ${ }^{\mathrm{a}, \mathrm{b}}$, Robert Kozol ${ }^{\mathrm{b}}$
}

\begin{abstract}
Complete colonic obstruction due to endometriosis is infrequently seen. Acute colonic obstruction, as presented in this case study, is an even more rare event with very few incidences having been reported in the literature. The signs and symptoms of such an event are very non-specific and may mimic the differential diagnosis of a malignant neoplasm. We present a case of a 34 year-old female who's clinical and radiographic course mimicked a neoplasm and it was not until the histopathological examination during surgery that the diagnosis of endometriosis was made. This report discusses the etiology, differential diagnosis, and surgical management of such a case
\end{abstract}

Keywords: Edometriosis; Colonic obstruction; Surgery; Neoplasm

\section{Introduction}

Endometriosis is defined as a chronic, benign, estrogen-dependent disorder characterized by the implantation of functional endometrial tissue in extra-uterine sites. These ectopic sites commonly include the structures in the pelvis, however endometriosis has been described in a diversity of organs and spaces in the body. The most common sites of ectopic endometrial implantation in decreasing order of frequency includes; the ovaries, rectal pouch, uterus, fallopian tubes, sigmoid colon, and appendix [1]. We present a rare case of

Manuscript accepted for publication June 7, 2012

${ }^{a}$ Nova Southeastern College of Osteopathic Medicine, 3301 College
Avenue, Fort Lauderdale-Davie, Florida 33314-7796, USA
${ }^{b}$ University of Miami- Miller School of Medicine, JFK Medical
Center5301 South Congress Avenue, Atlantis, FL 33462, USA
${ }^{\text {c} C o r n e l l ~ U n i v e r s t i y, ~} 410$ Thurston Ave Ithaca, NY 14850- 2488, USA
${ }^{d}$ Corresponding author: Elias Fakhoury, 2800 South Seacrest Blvd, Suite
200, Boynton Beach, Florida, USA. Email: fakhoury.elias@gmail.com

doi: $10.4021 / \mathrm{jcs} 44 \mathrm{w}$ complete colonic obstruction attributed to invasive endometriosis of the rectum whose clinical and radiographic course mimicked a malignant neoplasm.

\section{Case Report}

A 34-year-old African American female, with a past medical history significant for hypertension, and a surgical history significant for a hysterectomy (without oophorectomy) five years prior, presented to our teaching hospital with abdominal pain, nausea and vomiting. She had not had a bowel movement or experienced flatus for five days. She denied weight loss, anorexia, generalized weakness or any other constitutional symptoms prior the starting of her clinical presentation. The physical exam revealed abdominal distention, hyperactive bowl sounds, absence of guarding, and no palpable masses. A bimanual pelvic exam was performed but was not helpful due to the patient's body habitus. The digital rectal examination was normal. Initial laboratory studies including a $\mathrm{CBC}$, serum chemistries, and liver function test were unremarkable. A non-contrast computed- topography (CT) of the abdomen and pelvis performed in the emergency department revealed a pelvic mass that was encasing the recto-sigmoid junction and causing complete colonic obstruction (Fig. 1). An urgent diverting sigmoid loop colostomy was performed

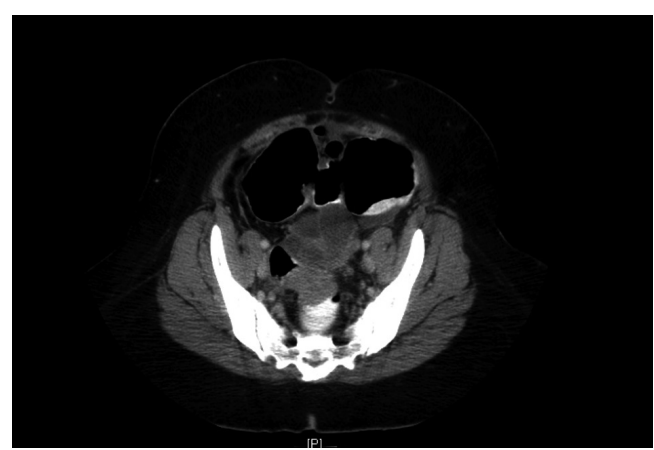

Figure 1. Non contrast CT of abdomen and pelvis showing a $3.0 \times 3.0 \mathrm{~cm}$, soft tissue density mass at the level of the sigmoid-rectal junction and an adjacent complex cystic mass measuring $5.5 \mathrm{~cm}$ in diameter. 


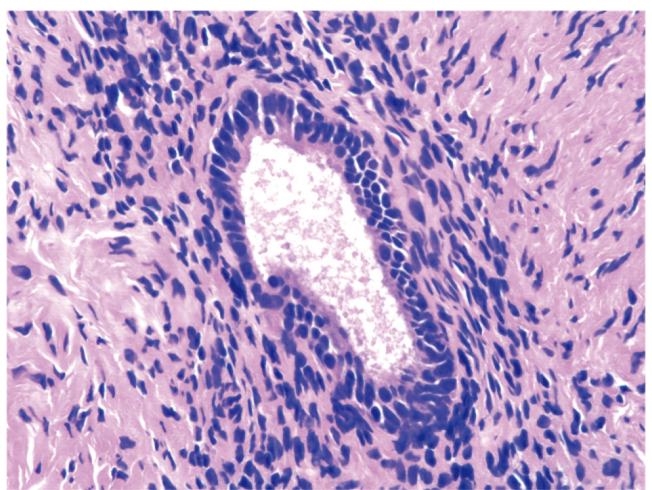

Figure 2. High powered image of irregular endometrial glands surrounded by dense stroma.

for decompression. Colonoscopy performed before and after the fecal diversion procedure ruled out adenocarcinoma of the colon but confirmed a complete mechanical obstruction at the recto-sigmoid junction. The differential diagnosis included ovarian carcinoma.

Due to delays on the part of the patient, the definitive operation occurred three months later. The plan was definitive resection of the pelvic mass and restoration of intestinal continuity. Upon accessing the pelvis, extensive fibrotic tissue adjacent to a non- delineated mass was found to be in intimate connection with the bladder, uterus, rectum and distal ileum. Resection of the specimen with clear distal rectal margins and elimination of the entire gross pelvic mass, which included the recto-sigmoid junction and the left ovary and fallopian tube, was achieved with great difficulty. The diverting colostomy was left in place as a Hartman's procedure. An anastamosis was not done because of contusion of the rectal stump. Intra-operative pathologic evaluations of the specimen revealed findings consistent with endometriosis with no indications of a malignant neoplasm (Fig. 2, 3). The patient had an un-eventful recovery and three months later underwent a colostomy reversal. She tolerated the procedure well and was discharged home on postoperative day five, after tolerating diet.

\section{Discussion}

The three most common causes of large bowel obstruction have been reported as being cancer, diverticulitis and volvulus [2]. Endometriosis affects $4-17 \%$ of menstruating women with a peak incidence in the third and fourth decade of life [3-5]. The gastrointestinal tract is involved in 3-34\% of these cases with the rectum and rectosigmoid junction accounting for $70-90 \%$ of all intestinal endometriosis cases $[4,6]$. The incidence of complete colonic obstruction due to endometriosis has not been estimated and is an exceedingly rare condition, in part due to the diameter of the colon and the

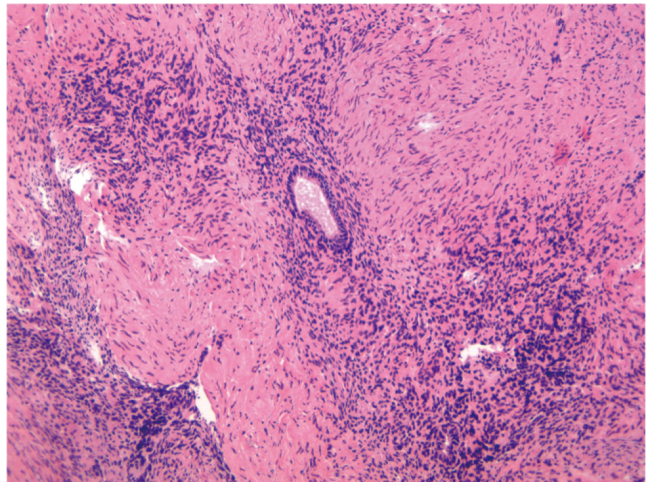

Figure 3. This image shows an elongated tubular structure surrounded by irregular stromal cells involving the muscularis propia of the bowel.

difficulty of encasing it.

The exact etiology of endometriosis remains unknown, however the three leading theories which are most widely accepted include: (1) retrograde menstruation; (2) coelomic metaplasia and (3) vascular or lymphatic spread. Even though endometriosis may be completely asymptomatic, the clinical presentation includes the signs and symptoms of; dysmenorrhea, abnormal bleeding, painful stooling, intestinal obstruction, and infertility. When symptomatic, patients may present with complaints that occur cyclically in association with menstruation.

The gold standard method for diagnosis of endometriosis is laparoscopic biopsy, although the diagnosis may be suspected on the basis of the clinical history. Imaging modalities such as CT, multislice CT, Magnetic Resonance Imaging (MRI), and rectal endoscopic ultrasonography have also proven to be useful [7]. The use of serum markers such as cancer antigen CA-125 (high sensitivity, low specificity) have gained interest and have proven to be helpful in the diagnosis [7].

The diagnosis of gastrointestinal endometriosis has proven to be more of a challenge because GI tract symptoms may mimic a wide variety of diseases, including irritable bowel syndrome, infectious diseases, ischemic enteritis, inflammatory bowel disease, and neoplasm [7]. GI endometriosis presents with abdominal pain, bouts of constipation and diarrhea, tenesmus, and rectal bleeding. In our case, a pre-operative diagnosis of endometriosis was not established as we suspected an extra colonic malignancy such as ovarian carcinoma. The true incidence of endometriosis causing bowel obstruction is unknown [8], although complete obstruction of the bowel lumen occurs in less then $1 \%$ of cases [9]. Our case demonstrates the difficulty of diagnosing and surgically treating colonic obstructions secondary to advanced endometriosis and its diverse manifestations.

The definitive treatment of endometriosis includes combination oral contraceptives, GnRH agonist, and progestins. 
Surgery is indicated for intestinal endometriosis leading to obstruction or bleeding when the symptoms fail to resolve or if a neoplasm cannot be excluded [7, 10].

\section{References}

1. Gustofson RL, Kim N, Liu S, Stratton P. Endometriosis and the appendix: a case series and comprehensive review of the literature. Fertil Steril. 2006;86(2):298-303.

2. Yantiss RK, Clement PB, Young RH. Endometriosis of the intestinal tract: a study of 44 cases of a disease that may cause diverse challenges in clinical and pathologic evaluation. Am J Surg Pathol. 2001;25(4):445-454.

3. Slesser AA, Sultan S, Kubba F, Sellu DP. Acute small bowel obstruction secondary to intestinal endometriosis, an elusive condition: a case report. World J Emerg Surg. 2010;5:27.

4. Pramateftakis MG, Psomas S, Kanellos D, Vrakas G, Roidos G, Makrantonakis A, Kanellos I. Large bowel obstruction due to endometriosis. Tech Coloproctol. 2010;14 Suppl 1:S87-89.
5. Bianchi A, Pulido L, Espin F, Hidalgo LA, Heredia A, Fantova MJ, Muns R, et al. [Intestinal endometriosis. Current status]. Cir Esp. 2007;81(4):170-176.

6. Fleisch MC, Xafis D, De Bruyne F, Hucke J, Bender HG, Dall P. Radical resection of invasive endometriosis with bowel or bladder involvement--long-term results. Eur J Obstet Gynecol Reprod Biol. 2005;123(2):224-229.

7. De Ceglie A, Bilardi C, Blanchi S, Picasso M, Di Muzio M, Trimarchi A, Conio M. Acute small bowel obstruction caused by endometriosis: a case report and review of the literature. World J Gastroenterol. 2008;14(21):34303434.

8. Paksoy M, Karabicak I, Ayan F, Aydogan F. Intestinal obstruction due to rectal endometriosis. Mt Sinai J Med. 2005;72(6):405-408.

9. de Bree E, Schoretsanitis G, Melissas J, Christodoulakis M, Tsiftsis D. Acute intestinal obstruction caused by endometriosis mimicking sigmoid carcinoma. Acta Gastroenterol Belg. 1998;61(3):376-378.

10. Lin YH, Kuo LJ, Chuang AY, Cheng TI, Hung CF. Extrapelvic endometriosis complicated with colonic obstruction. J Chin Med Assoc. 2006;69(1):47-50. 